\title{
Novel sheathless CE-MS interface as an original and powerful infusion platform for nanoESI study: from intact proteins to high molecular mass noncovalent complexes
}

Rabah Gahoual ${ }^{1}$, Jean-Marc Busnel ${ }^{2}$, Philippe Wolf ${ }^{3,4}$, Yannis Nicolas François *1, Emmanuelle Leize-Wagner ${ }^{1}$

${ }^{1}$ Laboratoire de Spectrométrie de Masse des Interactions et des Systèmes (LSMIS), CNRS - UMR 7140, Université de Strasbourg, Strasbourg, France. ${ }^{2}$ Beckman Coulter Inc., Brea, CA, United States. ${ }^{3}$ Architecture et Réactivité de l'ARN, Université de Strasbourg, Institut de Biologie Moléculaire et Cellulaire, Strasbourg, France. ${ }^{4}$ Plateforme Protéomique de l'Esplanade Université de Strasbourg, Institut de Biologie Moléculaire et Cellulaire, Strasbourg, France.

*Corresponding author phone: +33-3-68-85-16-41 ; email : yfrancois@unistra.fr 


\begin{abstract}
Development of nano electrospray (nanoESI) sources allowed to increase significantly the sensitivity which is often lacking when studying biological noncovalent assemblies. Yet the flow rate used to infuse the sample into the MS cannot be precisely controlled with nanoESI and robustness of the system could represent an issue. In this study we have used a sheathless capillary electrophoresis - mass spectrometry (CESI) prototype as a nanoESI infusion device. The hydrodynamic mobilization of the capillary content was characterized and the ability of the system to generate a stable electrospray under controlled flow rate conditions ranging from $4 \mathrm{~nL} / \mathrm{min}$ up to $900 \mathrm{~nL} / \mathrm{min}$ was demonstrated. We investigated the effect of the infusing flow rate on the detection of an intact model protein analyzed under native conditions. Results demonstrated a significant increase in sensitivity of 46 fold and a signal-to-noise ratio improvement of nearly 5 fold when using infusing flow rate from 456.9 down to $13.7 \mathrm{~nL} / \mathrm{min}$. The CESI prototype was further used to detect successfully the $\beta$ ring homodimer in its native conformation. Obtained results were compared to those achieved with conventional ESI. Intensity signals were increased by a factor of 5 while sample consumption decreased 80 times. $\beta$ ring complexed with the P14 peptide was also studied. Finally the CESI interface was used to observe the quaternary structure of native hemocyanins from Carcinus maenas crabs, this high molecular complex coexisting under various degrees of complexation resulting in masses ranging from $445 \mathrm{kDa}$ to $1.34 \mathrm{MDa}$.
\end{abstract}

Keywords : sheathless capillary electrophoresis, mass spectrometry, noncovalent complexes

\author{
ABBREVIATIONS \\ MS Mass spectrometry \\ ESI Electrospray ionization \\ nanoESI Nano electrospray ionization \\ CESI Sheathless capillary electrophoresis \\ CE Capillary electrophoresis \\ HF Hydrofluoric acid \\ BGE Background electrolyte \\ IPTG $\quad \beta$-D-1-thiogalactopyranoside
}

\title{
INTRODUCTION
}

Structural determination of intact proteins and protein complexes has a major contribution into understanding fundamental biochemical pathways. Noncovalent 
interactions are decisive for numerous kinds of in vivo biochemical processes as assemblies of several proteins generally allow specific recognition, binding or transport. Mass spectrometry (MS) since the development of electrospray ionization (ESI) has grown into a major technique to study noncovalent interactions [1-3]. Indeed ESI is a soft ionization technique which allows transfer of noncovalent protein complexes to the gas phase without losing its quaternary structure [4]. MS is able to provide access to structural information [5], stoichiometry [6] and binding constant of noncovalent complexes [7]. In order to detect native complexes by MS it is mandatory to use aqueous buffers which do not disrupt the quaternary structure of the protein or the complex. Additionally, used buffers need to be relatively volatile enable ESI ionization / desorption processes. Ammonium acetate is one of the most commonly used buffers as it fulfills those requirements [8]. Salt concentrations necessary to maintain the complex in native conditions may vary from $20 \mathrm{mM}$ to $100 \mathrm{mM}$ or even higher. Such levels of salt concentration are quite significant and as a direct consequence ESI-MS in native conditions usually results in poor signal intensity and sensitivity mainly due to ion suppression effect [9-10]. A typical alternative to enhance signal intensity is to use a higher sample concentration (superior to $10 \mu \mathrm{M}$ ), however such conditions risk to favor nonspecific interactions and protein aggregation. The total volume of sample required to perform an experiment is also an important issue. In the context of the study of noncovalent complexes by classical ESI, the volume of sample to realize one acquisition is usually in the range of one hundred microliters. Such important volume may be unfavorable for studying biological samples which are only available in scarce amounts. Even if classical ESI has given the possibility to observe noncovalent edifices by MS, it was necessary to improve signal-to-noise 
ratio while concomitantly reducing sample consumption to realize the full potential of MS-based studies in this field.

To address both issues, Wilm and Mann introduced in 1994 a nano electrospray ionization source (nanoESI) causing a major advance in mass spectrometry [11]. With nanoESI, the sample is introduced in the MS through a tapered capillary with reduced internal diameter (from 1 to $5 \mu \mathrm{m}$ ). Such device being compatible with low flow rates (typically a few dozens $\mathrm{nL} / \mathrm{min}$ ) it allows to significantly lower the volumes of infused sample while maintaining a similar acquisition time. In nanoESI smaller droplets are produced increasing sensitivity and reducing ion suppression by improving analyte ionization efficiency as they facilitate Rayleigh divisions throughout the desolvation process. Using nanoESI they demonstrated that the MS detection of a synthetic peptide at a concentration of $1 \mu \mathrm{M}$ in $100 \mathrm{mM} \mathrm{NaCl}$ [12]. Thanks to the significant reduction in flow rates as compared to classical ESI, sample consumption was reduced to be at least 2 orders of magnitude in nanoESI. Typically, a nanoESI experiment is performed using a borosilicate glass capillary pulled in order to reduce the internal diameter of its extremity to ensure good compatibility with low flow rates. Capillary tip is then gold plated allowing grounding. Sample is introduced in the capillary and nanoESI is initiated by applying a potential between the capillary and the MS inlet [12]. Note that no pumping system is used to introduce the sample into the source, therefore infusion cannot be precisely controlled and flow rate robustness may be an issue. Though technical developments have been realized allowing automated nanoESI to be performed using chip-based automated infusion systems [13]. The robustness of such system may be problematic especially in the case of samples containing relatively high ionic strength buffers typically used to maintain noncovalent 
complexes. Indeed, to the best of our knowledge such automated systems have not yet been used to study high molecular mass noncovalent assemblies (superior to $250 \mathrm{kDa}$ ) [14]. That observation limits unfortunately the use of automated systems for high molecular mass and complex assemblies which have been subject to intensive research work in the recent years [5].

Recently a novel interface (referred as CESI) allowing the hyphenation of capillary electrophoresis (CE) with ESI-MS in a single dynamic process within the same device has been developed by Beckman Coulter. It is based on a design previously described by Moini [15]. This sheathless interface uses a fused silica capillary whose outlet has been etched using hydrofluoric acid (HF) making it porous to the electrical transport of small ions. Detailed description of this interface has been given by Haselberg et al. [16]. It has already been used to perform successful CE-MS experiment in proteomics [17-23], metabolomics [24], intact proteins $[16,25-26]$ demonstrating drastically increased sensitivity compared to sheath-liquid CE-MS due to low operating flow rates lower than $10 \mathrm{~nL} / \mathrm{min}$ to about $100 \mathrm{~nL} / \mathrm{min}$

After having studied the suitability of the CESI prototype to be used as a nanoESI infusion device the aim was to assess its potential as compared to either conventional ESI or classical approaches to nanoESI under very demanding conditions such as native MS of large noncovalent complexes. To this end, parameters such as ease of use, sensitivity gain, robustness and sample consumption have been considered. Electromigration separation was not involved in this work properly but the intrinsic characteristics of this system (miniaturized aspect, low infusion flow rate) and the possibility to realize accurate hydrodynamic infusion were used to infuse the sample in the source and generate fully controlled nanoESI 
experiments. Then, a model protein, $\beta$-ring, was studied in native conditions with both CESI and conventional ESI to determine the ability of the CESI interface to maintain noncovalent complexes such as protein-protein and protein-ligand interactions. Finally as a last step, a biological high molecular mass complex was successfully observed using the CESI prototype through the successful detection of the considered complex assembly, maintained by weak interactions having a molecular mass superior to $1.3 \mathrm{MDa}$, it was demonstrated that CESI is well suited for such studies. The compatibility of the system with very low flow rates and its robustness enabled the achievement of producing high quality mass spectra and accurate mass determination while consuming very limited amounts of samples.

\section{MATERIALS AND METHODS}

Reagents

Myoglobin and all chemicals used were of analytical grade or high purity grade and purchased from Sigma-Aldrich (Saint Louis, MO, USA). Water used to prepare buffers and sample solutions was obtained using an ELGA purelab UHQ PS water purification system (Bucks, UK).Desalting steps were performed using Amicon Ultra $10 \mathrm{~K}$ centrifugal filters purchase from Millipore (Molsheim, France). $\beta$-ring samples were kindly provided by Dominique Burnouff group (IBMC, Strasbourg, France), Carcinus maenas hemolymph samples were supplied by Frank Zal group (Roscoff, France).

Production of the $\beta$ ring and P14 peptide 
The Pseudomonas dnaN gene was cloned into pET15b plasmid (Invitrogen) using standard protocols. The resulting N-tagged protein was expressed in BL21 Pseudomonas cells after isopropyl $\beta$-D-1-thiogalactopyranoside (IPTG) induction $(0.1 \mathrm{mM})$ at $28^{\circ} \mathrm{C}$. The $\beta$ protein fraction was first enriched on a Ni-NTA column, eluted with an histidine step $(300 \mathrm{mM})$ and further purified on a MonoQ column in a buffer containing $20 \mathrm{mM}$ Tris $\mathrm{HCl}(\mathrm{pH} 7.5), 0.5 \mathrm{mM}$ EDTA and $10 \%$ glycerol, using a gradient from 0 to $0,5 \mathrm{M} \mathrm{NaCl}$. The pure protein was dialyzed and concentrated (around $300 \mu \mathrm{M}$ ) against a buffer containing 20mM Tris $\mathrm{HCl}(\mathrm{pH} 7.5)$, $0.5 \mathrm{mM}$ EDTA and $10 \%$ glycerol. P14 peptide (Ac-Q Cha DL diClF, M= $785 \mathrm{Da}$ ) was synthetized using a procedure described previously by Wolff et al. [27].

Hemocyanin sample preparation

Hemolymph sample collection and preparation were adapted from Sanglier et al. [6]. Prior to use, frozen Carcinus maenas hemolymph samples were thawed at $4^{\circ} \mathrm{C}$ and further centrifuged at $12,000 \mathrm{rpm}$ during $15 \mathrm{~min}$. The obtained supernatant was collected and considered for a further treatment. Collected supernatants were desalted using Amicon centrifugal filters (cut off $=10,000$ Da) in $20 \mathrm{mM}$ ammonium acetate $(\mathrm{pH}=6.8)$. Nine desalting steps were performed at $4^{\circ} \mathrm{C}$ and $14,000 \mathrm{~g}$ for $20 \mathrm{~min}$ each step. Ammonium acetate allows proteins native structure to be preserved and is compatible to ESI-MS.

\section{$\mathrm{CE}$ as infusion platform}

The infusion experiments were carried out with a PA 800 plus capillary electrophoresis (CE) system from Beckman Coulter (Brea, CA) equipped with a temperature controlled autosampler and a power supply able to deliver up to $30 \mathrm{kV}$. 
Prototype fused-silica capillaries (total length $100 \mathrm{~cm} ; 30 \mu \mathrm{m}$ i.d.) whose outlet end (about $3 \mathrm{~cm}$ ) was etched with hydrofluoric acid were used for all related CESI experiments and initially provided by Beckman Coulter (Brea, CA, USA). It is certainly noteworthy to precise here that the inner lumen of these capillaries is not tapered and presents an i.d. of $30 \mu \mathrm{m}$ throughout its entire length. New capillaries were initially conditioned by flushing them for $10 \mathrm{~min}$ with $\mathrm{MeOH}, 10 \mathrm{~min}$ with $0.1 \mathrm{M}$ sodium hydroxide, $10 \mathrm{~min}$ with $0.1 \mathrm{M}$ hydrochloric acid, water for $20 \mathrm{~min}$ and finally for 10 min with background electrolyte (BGE) each flushing step being conducted at $75 \mathrm{psi}(5.17 \mathrm{bar})$. BGEs used is ammonium acetate $20 \mathrm{mM}$ or $50 \mathrm{mM}$ depending on the sample at physiological $\mathrm{pH}$ ( $\mathrm{pH}$ ranging from 6.7 to 7). Hydrodynamic injection provided by the CE pressure delivery system was used to infuse the sample through the CESI prototype.

Mass Spectrometry and data analysis

For sheathless CE-ESI-MS experiments the CE system was coupled to a microTOFQ II (Bruker Daltonics, Bremen, Germany) mass spectrometer for general characterization and protein-ligand applications and to a microTOF (Bruker Daltonics, Bremen, Germany) mass spectrometer for analysis of the hemocyanin samples. MS transfer parameters were optimized using the actual sample directly infused via the CE system using a pressure of 5 psi (340 mbar). MS parameters were optimized so that heavy ions could be properly transferred to the TOF analyzer while avoiding fragmentation. In the case of the microTOF-Q MS, ion funnels were set at values of $300 \mathrm{Vpp}$ and $400 \mathrm{Vpp}$. When using the microTOF MS capillary exit was set at a value of $140 \mathrm{~V}$. The electrospray voltage (capillary voltage) was typically ranging from -1.2 to $-1.8 \mathrm{kV}$ depending on the composition of the buffer. 
Thanks to the design of the CESI interface, no nebullizer gas is required. Dry gas was set at $1.5 \mathrm{~L} / \mathrm{min}$ and source temperature at $180^{\circ} \mathrm{C}$.

Evaluation of flow rate and infusion stability

While the capillary was initially filled with $10 \%$ acetic acid, a sample of myoglobin at a concentration of $1 \mu \mathrm{M}(16.95 \mathrm{ng} / \mu \mathrm{L})$ in $10 \%$ acetic acid was infused through the $\mathrm{CE}$ system using different pressure (from 20 to $0.4 \mathrm{psi}$ ). To estimate infusion flow rates, time necessary to the sample to fill the entire capillary was measured following the MS signal. The experiment was repeated in triplicate for each pressure.

Evaluation of CESI sensitivity Myoglobin sample at a concentration of $250 \mathrm{nM}$ in $20 \mathrm{mM}$ ammonium acetate (native conditions) was infused through the CE system using different pressure (from 100 to 0.4 psi). For each pressure, sample mass spectrum was recorded for 4 min. An average mass spectrum was then generated using the last $3 \mathrm{~min}$ (first minute was excluded to ensure flow rate signal stabilization). Signal intensity and sensitivity (amount of sample introduced to the MS) were plotted against infusion flow rate. The full experiment was replicated four times.

\section{RESULTS AND DISCUSSION}

Evaluation of CESI interface as a nanospray emitter. As a preliminary experiment, the CESI interface was studied regarding the range of infusion flow rate accessible while maintaining a stable electrospray. CE experiments are usually 
performed in a reduced volume of a few nanoliters due to the total capillary volume not exceeding a few microliter. Therefore CE systems are designed to accurately execute injection of small volumes of sample $(<1 \mu \mathrm{L}$ injected), the pressure system can also be used to infuse a sample solution through the capillary at very low flow rates in a stable and robust way. Hydrodynamic injection is made by applying a pressure over the contents of a vial located at the inlet of the capillary. While flow rates can theoretically be calculated using the Hagen-Poiseuille equation (1), it was important to experimentally measure the actual flow rates under the considered nanoESI conditions, such measurements allowing to take into account the viscosity of the sample solution and further the potential suction effect induced by the ESI process itself. Indeed the capillary tip is positioned at a distance of about $2 \mathrm{~mm}$ from the MS entrance, this proximity requires to determine if at low flow rates the potential applied in the source does not create a suction effect of the sample similar to those observed with borosilicate glass capillaries in nanoESI [12]. That effect could potentially disrupt the pressure applied by the CE making flow rate control impossible.

Myoglobin in $10 \%$ acetic acid was used as a model protein to evaluate flow rates. Sample was injected through the capillary at a given pressure. The capillary, initially only filled with buffer, is progressively filled with the sample until the myoglobin is detected by the MS. The time necessary to record MS signal corresponding to myoglobin (detection time) is obtained and considered for flow rate calculation. Viscosity of the buffer was then calculated using the HagenPoiseuille (1) equation. Flow rates were calculated from the detection time and the capillary total volume. 


$$
\eta=\frac{d_{c}{ }^{2} \Delta P t}{32 L^{2}}
$$

Where $d_{c}$ is the capillary internal diameter, $\Delta P$ the applied pressure, $t$ the detection time, $L$ the total length of the capillary.

The measured flow rate were then plotted as a function of the pressure used for mobilization (Fig 1). Results obtained show the capacity of the system to maintain a stable electrospray with a pressure as low as 0.4 psi corresponding to a flow rate of $3.8 \mathrm{~nL} / \mathrm{min}$. Moreover to the possibility to realize sample infusion at very low flow rates, the system has also demonstrated a significant robustness as the relative standard deviation calculated amongst the repetitions is in every case inferior to 1.1\%. Additionally, the linear regression showed a correlation coefficient superior to 0.999 . The proportional relationship between the applied pressure and the flow rate clearly illustrates the fact that no significant suction effect is observed under the tested flow rates, meaning the injecting flow rate is only induced by the CE hydrodynamic injection system. This trend, in contrast to other nanoESI source, to prevent sample aspiration due to the electrical field could be explained by the internal diameter of the capillary. Indeed the use of a capillary having an internal diameter of $30 \mu \mathrm{m}$ increases the surface tension of the liquid preventing it from being pulled out of the capillary by the electrical field [11]. In addition the CE system is able to deliver fully controlled hydrodynamic injections even at infusion flow rates as low as $4 \mathrm{~nL} / \mathrm{min}$. 


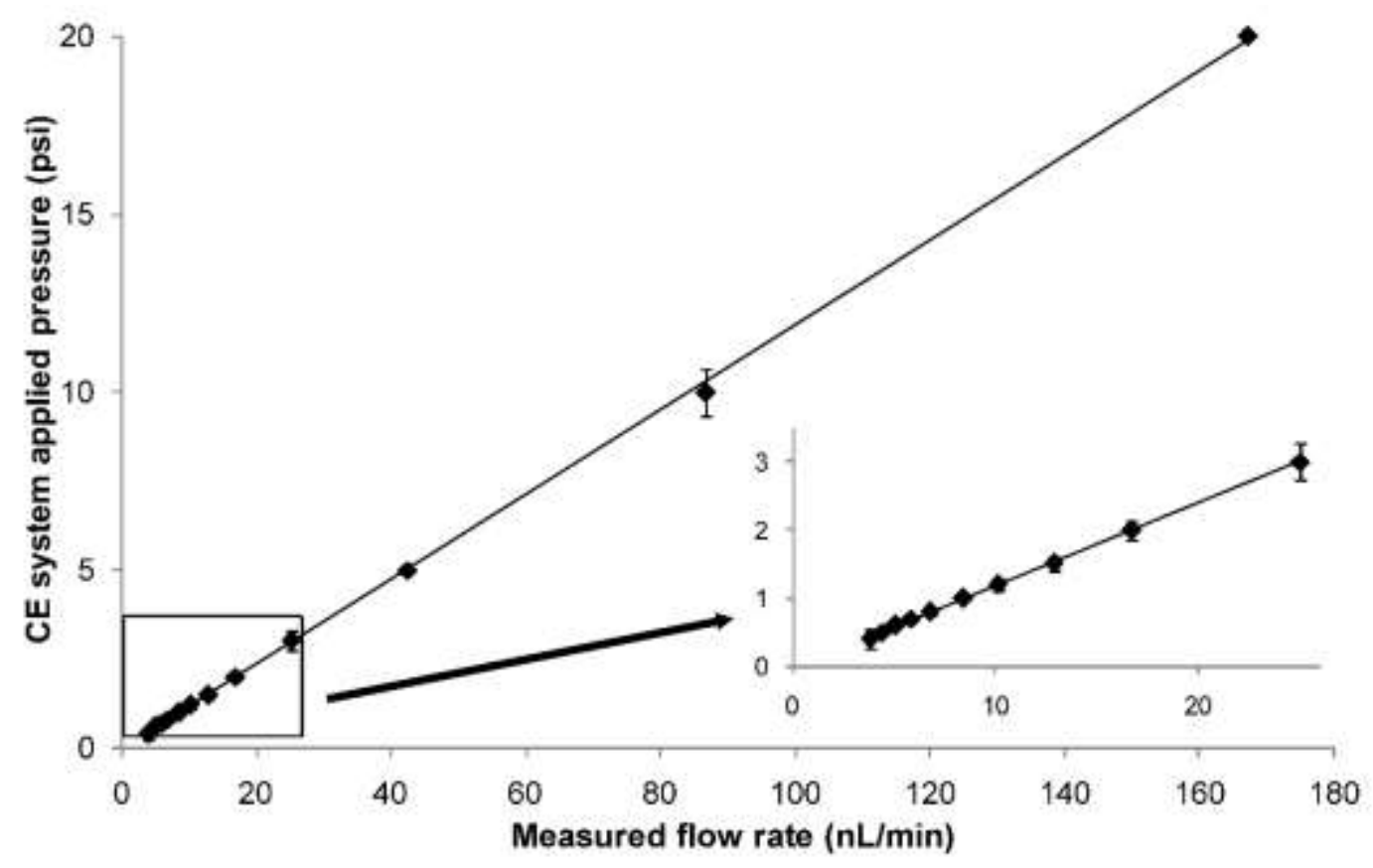

Fig 1. Evolution of the infusing flow rate as a function of the applied pressure. Experimental conditions : bare fused silica capillary with porous tip, total length 92 cm $(30 \mu \mathrm{m}$ i.d., $150 \mu \mathrm{m}$ o.d.). Sample : myoglobin $1 \mu \mathrm{M}$ (in 10\% AA). Mass spectrometry : capillary voltage $-1400 \mathrm{~V}$, considered $\mathrm{m} / \mathrm{z} 848.94$.

As a second step to characterize the electrospray generated through the CESI interface, the influence of flow rate on sensitivity under native conditions was studied. Effect of flow rate on sensitivity in ESI has already been discussed [1828]. Under conventional ESI flow rates, native MS usually lacks sensitivity as it is intensively subject to ion suppression and as such typically benefits from the nanoESI regime. Using the CESI prototype as an infusion device, sensitivity was evaluated using myoglobin at a rather low concentration $(250 \mathrm{nM})$ in native conditions (20 mM ammonium acetate, $\mathrm{pH} 6.8$ as buffer). The sample was infused into the MS through the system at different flow rates ranging from 3.8 to 913 $\mathrm{nL} / \mathrm{min}$. Sensitivity defined as the ratio of MS spectrum intensity to the quantity of introduced sample in the source has then been plotted against flow rate (Fig 2). 


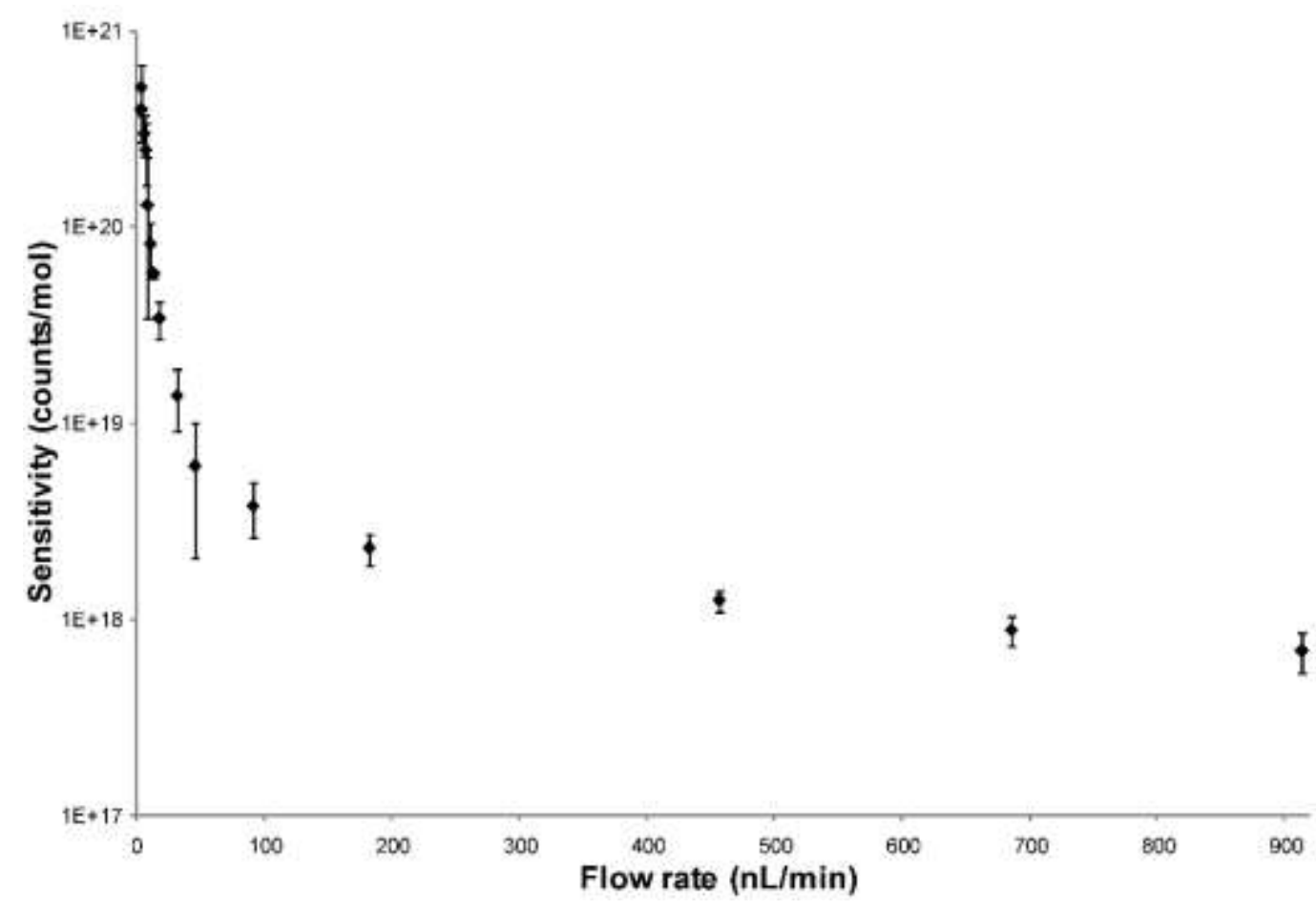

Fig 2. Evolution of signal sensitivity (logarithmic scale) as a function of the infusion flow rate in native conditions. Experimental conditions : bare fused silica capillary with porous tip, total length $92 \mathrm{~cm}(30 \mu \mathrm{m}$ i.d., $150 \mu \mathrm{m}$ o.d.). Sample : myoglobin $250 \mathrm{nM}$ (in $20 \mathrm{mM}$ ammonium acetate). Mass spectrometry : capillary voltage $1400 \mathrm{~V}$, considered $\mathrm{m} / \mathrm{z} 2196.8$.

Results collected show a significant increase of the sensitivity as the infusion flow rate is reduced. As a consequence, sensitivity was increased by a factor of 46 while reducing the infusion flow rates from 456.9 to $13.7 \mathrm{~nL} / \mathrm{min}$. Even greater values have been obtained for lower flow rates. Signal-to-noise ratio is also significantly enhanced with respective values of 189.5 at $456.9 \mathrm{~nL} / \mathrm{min}$ and 920.4 at $13.7 \mathrm{~nL} / \mathrm{min}$ (data not shown) demonstrating reduction of the ion suppression effect. Such improvement in sensitivity, even for unfavored conditions as native MS is a huge asset in the perspective of noncovalent complexes study. In agreement with data presented elsewhere, an increase in sensitivity was observed when decreasing the infusion flow rate [18]. In the referenced work, it was demonstrated that the described interface provided a stable spray at flow rates as low as below $10 \mathrm{~nL} / \mathrm{min}$, 
thereby enabling to work not only in the concentration-sensitive region of ESI-MS but also in the mass-sensitive one. As a direct consequence, additionally to provide higher ionization efficiencies, a lower ion suppression is expected. In the context of the presented study, this directly impacts the compatibility of the system to work under native conditions. This is well exhibited by Figure 2 where an increase in sensitivity is observed when decreasing the flowrate. Within the mass-sensitive region where the signal is proportional to the amount of molecules injected per unit of time, the sensitivity should theoretically remain constant. Nevertheless, it can be observed on Figure 2 that the sensitivity keeps increasing when decreasing the flow rate. The related experiment being achieved under native conditions, we believe that this phenomenon is related to the ion suppression whose magnitude decreases with the flow rate.

Other nanoESI source uses capillaries having internal diameter ranging from 1 to $10 \mu \mathrm{m}$. With the CESI system the use of a $30 \mu \mathrm{m}$ i.d. capillary allows to increase drastically the sensitivity as well. This could be explained by the fact that the flow rate is fully controlled. Mann and Wilm modelized the droplets emission radius from the Bernoulli equation; they demonstrated that this radius was proportional to $2 / 3$ power of the flow rate [11], meaning that the droplet emission radius may be more influenced by the flow rate than the internal diameter, the ability of the system to operate at a controlled nano flow rate should not increase the emission radius allowing to maximize ionization by still focusing the droplet emission zone towards the MS inlet. Flow rate measures and sensitivities were repeated several times, they demonstrated the repeatability of the method which is showed in Figure 2 by the relatively low deviation recorded from one experiment to another. 
Those results clearly demonstrates the capacity of the considered CESI-MS system to generate a so-called nano electrospray with the capabilities to have a full control on flow rate over an extended range going from around $4 \mathrm{~nL} / \mathrm{min}$ to 500 $\mathrm{nL} / \mathrm{min}$ and even more. In native conditions, sensitivities could be increased by a factor over 46 while improving as well signal-to-noise ratio. Those results clearly indicate the possibility to enhance sensitivity for detecting noncovalent complexes using this system.

\section{Protein-ligand complexes characterization by CESI-MS and comparison to} conventional ESI-MS. Study of biological protein-protein and protein-ligand noncovalent interactions are important and subject to intensive research as they are involved in many in vivo biochemical processes. Results presented previously illustrate the ability of the CESI prototype to be operated at very low flow rates thereby drastically increase sensitivity. The suitability of the system for noncovalent complexes study was evaluated using a model protein. Pseudomonas processivity factor, also referred as $\beta$-ring is a homodimer of about $85.45 \mathrm{kDa}$ that encircles the bacterial DNA and anchors the replicative DNA polymerase, which consequently acquires its high processivity. The ring is also a landing platform for many other enzymes which contribute to DNA metabolism. A sample of $\beta$-ring in $50 \mathrm{mM}$ ammonium acetate $(\mathrm{pH}$ 7.0) was infused into the MS using either a conventional ESI source operating at a flow rate of $4 \mu \mathrm{L} / \mathrm{min}$ or the CESI prototype operating at $50 \mathrm{~nL} / \mathrm{min}$. MS transfer parameters were identical. Mass spectra obtained are presented in Figure 3. The analytes detected have deconvoluted masses of $85,457.2 \pm 1.7 \mathrm{Da}$ and $85,457.6 \pm 1.8$ Da respectively using the conventional ESI source and the CESI interface (expected mass 85,456.0 Da). Those results allowed 
the accurate detection of the protein in its native homodimeric form with both sources
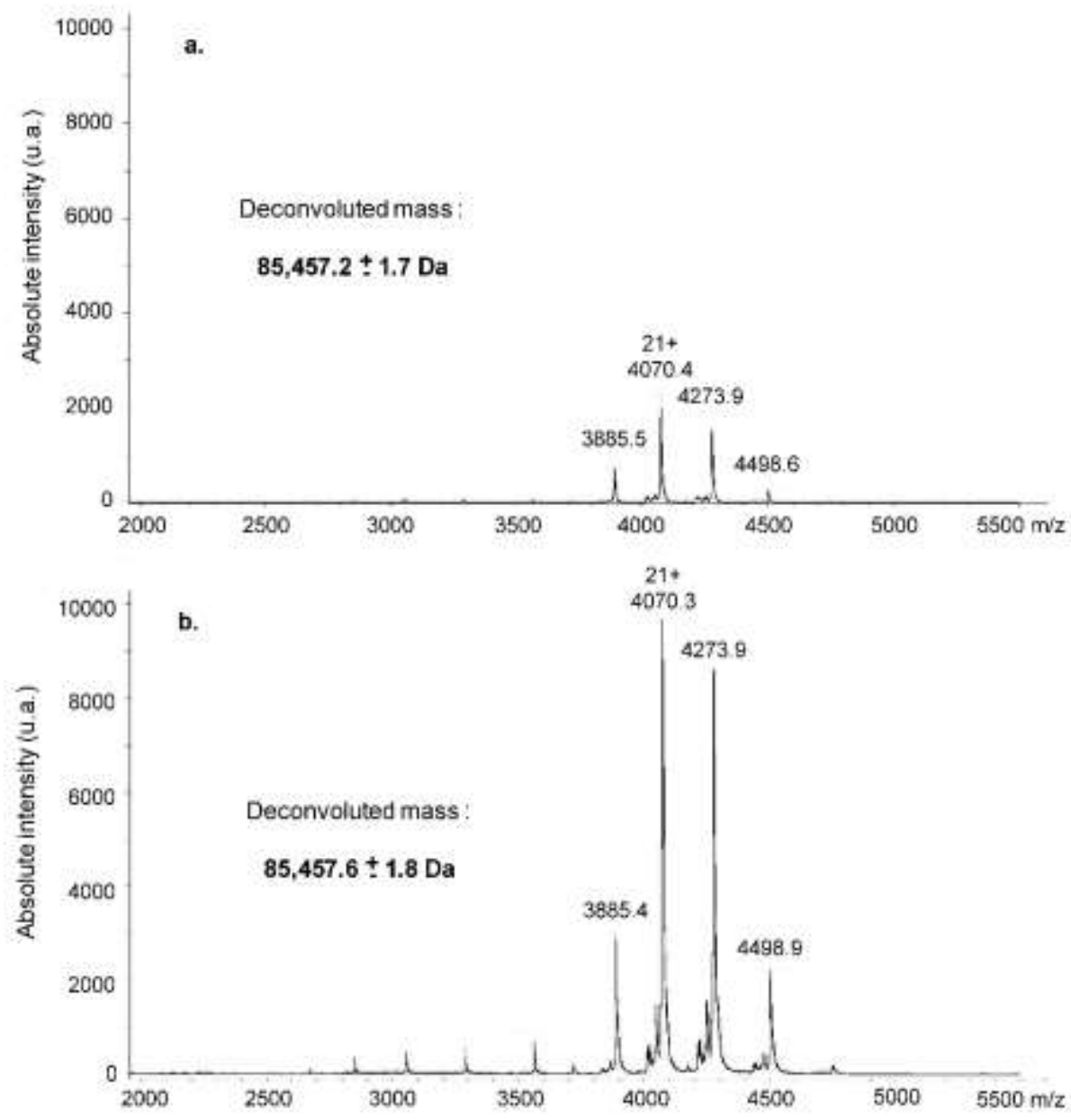

Fig 3. Comparison of $\beta$ ring mass spectra in native conditions by A) ESI-MS and B) CESI-MS. Experimental conditions : $\beta$ ring sample $(1 \mu \mathrm{M}$ in $50 \mathrm{mM}$ ammonium acetate) ; A) conventional ESI source, flow rate $4 \mu \mathrm{L} / \mathbf{m i n}$, capillary voltage $4500 \mathrm{~V}$, dry gas $4 \mathrm{~L} / \mathrm{min}$, source temperature $180^{\circ} \mathrm{C}$; B) bare fused silica capillary with porous tip $(30 \mu \mathrm{m}$ i.d.), flow rate $50 \mathrm{~nL} / \mathbf{m i n}$, capillary voltage $-1500 \mathrm{~V}$, dry gas $1.5 \mathrm{~L} / \mathrm{min}$, source temperature $180^{\circ} \mathrm{C}$.

As compared to conventional ESI, in term of signal intensities, mass spectra obtained via CESI exhibited, for the main peak, an intensity roughly 5 times higher. With both sources, the same 4 charge states comprised between $(20+)$ and $(23+)$ 
could be resolved allowing intact protein mass determination with a satisfying residue inferior to $2 \mathrm{Da}$.

As compared to ESI, the quantity of $\beta$-ring sample used to complete an experiment was reduced by a factor of 80 while using the CESI prototype. The total volume injected to the source was only $500 \mathrm{~nL}$, this low value is clearly an advantage for noncovalent complexes mass analysis only available in low amounts as it allows to significantly increase the acquisition time to enhance resolution.

The study of noncovalent complexes induces the mass analysis of protein-ligand mixture at different concentrations to determine for example the stoichiometry or the binding constant of a given complex [13]. $\beta$-ring is an interesting model as all the partners of the ring interact with a same binding pocket located on each monomer [29]. In this work, the ligand chosen was the P14 peptide which has a high affinity for the binding pocket of the $\beta$-ring. The CESI prototype was then tested using a mixture of $\beta$-ring and P14 peptide in ammonium acetate buffer (see material and methods). After a 5 minutes incubation period, the mixture was infused into the MS at a flow rate of $20 \mathrm{~nL} / \mathrm{min}$. The mass spectrum obtained presented in Figure 4 exhibits three different envelope. The first one gives a deconvoluted mass of $85,456.5 \pm 1.1$ Da corresponding to the $\beta$-ring homodimer (expected mass 85,456.0 Da). The second profile has a deconvoluted mass of 86,242.5 $\pm 1.5 \mathrm{Da}$, meaning a mass difference of $785.8 \mathrm{Da}$ as compared to the $\beta$-ring homodimer profil (expected mass 85,241.0 Da). This difference corresponds to the mass of the P14 peptide. Therefore the second profile is associated to the noncovalent complex between the $\beta$-ring homodimer and one P14 peptide. The last profile presenting a deconvoluted mass of $87,028.3 \pm 0.8 \mathrm{Da}$, it corresponds to the complex between the $\beta$-ring homodimer and two P14 peptides (expected mass 87,026.0 Da). 


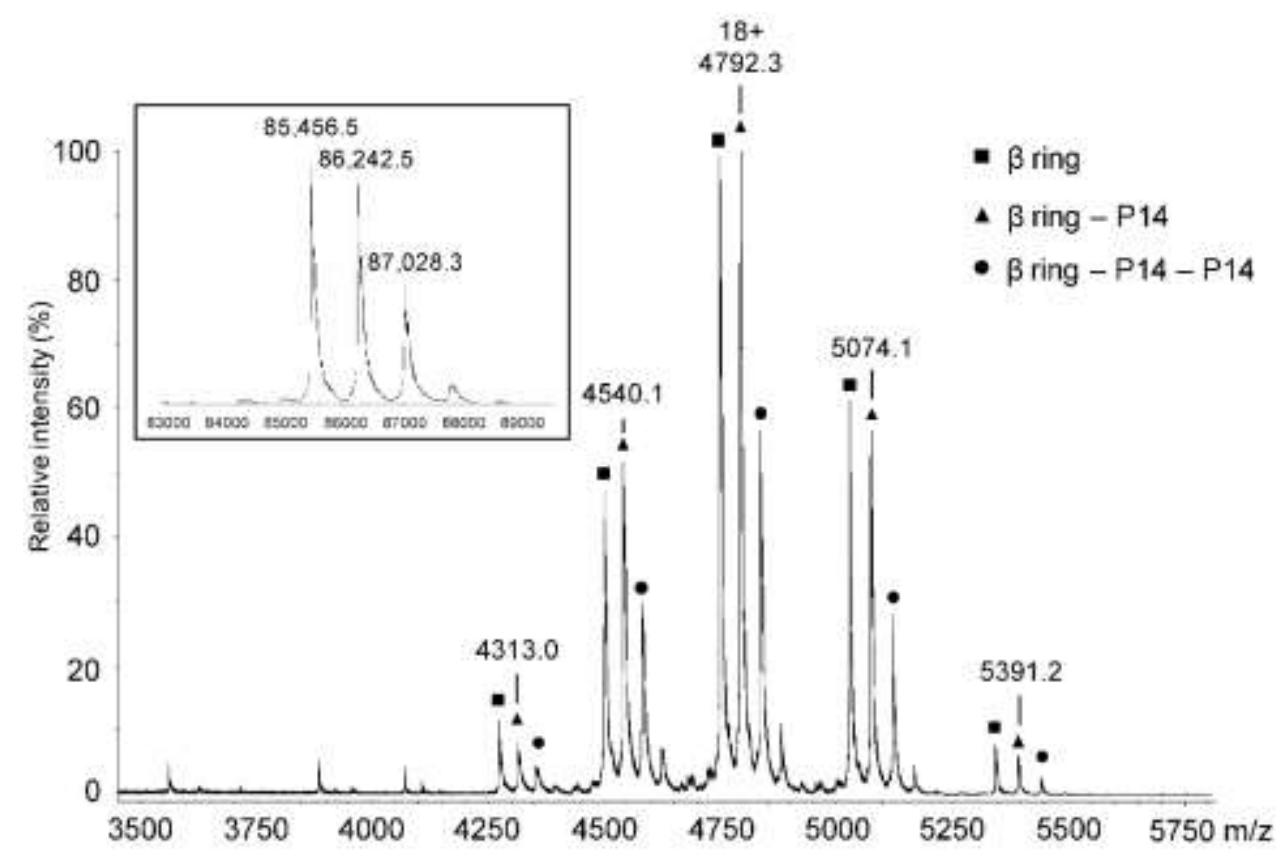

Fig 4. $\beta$ ring and $\mathrm{P} 14$ peptide mixture raw and deconvoluted mass spectra in native conditions. Experimental conditions : $\beta$ ring $0.3 \mu \mathrm{M}$ with $0.11 \mu \mathrm{M}$ P14 peptide (in $50 \mathrm{mM}$ ammonium acetate-1\% DMSO) ; bare fused silica capillary with porous tip (30 $\mu \mathrm{m}$ i.d.), flow rate $20 \mathrm{~nL} / \mathrm{min}$, capillary voltage $-1600 \mathrm{~V}$, dry gas $1.5 \mathrm{~L} / \mathrm{min}$, source temperature $180^{\circ} \mathrm{C}$.

Along with the protein homodimer naturally formed in native conditions, two different protein-ligand complexes could be successfully resolved. The CESI prototype allowed to operate with reduced ion suppression phenomena, resulting in a significant signal-to-noise ratio value of 138.7 , proper signal intensity and detection of several charge states. Also minimizing salt effect has for consequences to enable accurate detection of three different complexes even if those complexes have masses superior to $85 \mathrm{kDa}$ and mostly $\mathrm{m} / \mathrm{z}$ differences of only a few tens units. The infusion flow rate, reduced to $20 \mathrm{~nL} / \mathrm{min}$ was again fully controlled by the $\mathrm{CE}$ system, thereby stabilizing the MS signal and increasing the robustness of the system. In this case, for a 10 min acquisition, only $200 \mathrm{~nL}$ were needed to acquire the presented mass spectrum (Fig 4) and much longer acquisition could have been 
performed with less than one microliter. During all experiments no clogging of the capillary could be observed. After each acquisition the capillary was flushed with various buffer for several minutes and could be reused to infuse another sample without experimenting any cross-contamination. As compared to borosilicate capillaries used in conventional nanoESI which have internal diameter ranging from 1 to $5 \mu \mathrm{m}$, the internal diameter of the capillaries used in CESI is $30 \mu \mathrm{m}$, this difference significantly decreasing the possible occurrence of clogging, which is a very valuable advantage in this field.

By the mean of this experiment the system has been proven to be robust and easily operated. Additionally, a single capillary could be used to perform several of infusion under denaturing or native conditions.

High molecular mass noncovalent complexes. Along with the development of biological noncovalent complexes study by MS, researches have been continuously driven toward bigger and more complex assemblies. Such assemblies are usually available in low quantities and require a buffer heavily concentrated in salt to increase ionic strength and prevent dissociation, the ESI ionization process further allowing the conservation of assemblies even if maintained by quite weak interaction and this regardless their molecular mass. Considering that time of flight mass spectrometry (TOF-MS) has virtually an unlimited mass range analyzing ability, the detection of a wide range of noncovalent assemblies is possible even those having molecular masses of several megadaltons [30]. In order to confirm the capacity of the system to analyze very high molecular mass noncovalent complexes, a sample of hemocyanin was studied. Hemocyanin is a protein present in mollusks and arthropods hemolymph, and is involved in oxygen transport. One particularity of this protein is that it may contain one or several hexamers whose subunits have 
a mass approximately $75 \mathrm{kDa}$ [6]. Elements composing hemocyanin are maintained by weak noncovalent interactions. For this reason those complexes are usually difficult to observe in ESI-MS.

Hemocyanin directly extracted from Carcinus maenas hemolymph was analyzed using the considered CESI-MS system. In this case the sample was infused into the MS using a flow rate of $10 \mathrm{~nL} / \mathrm{min}$ in order to maximize the enhancement of sensitivity. A typical mass spectrum obtained is presented in Figure 5. It exhibits three different profiles. The first one detected in the range of $10600-11800 \mathrm{~m} / \mathrm{z}$ gives a deconvoluted mass of 445,242 $\pm 33 \mathrm{Da}$ and corresponds to the hexameric form of hemocyanin. The second profile detected between 13600 and $15550 \mathrm{~m} / \mathrm{z}$ provides a deconvoluted mass of $899,496 \pm 505 \mathrm{Da}$ which corresponds to the dodecamer form of hemocyanin. The last one detected in the range 16100-17900 has a deconvoluted mass of $1,337,489 \pm 456 \mathrm{Da}$ and can therefore be attributed to the octodecamer form of the protein. Mass measure obtained with the CESI-MS system are in complete agreement with the values described in the literature [6]. Also the standard deviation on measured masses obtained in this work demonstrate the sensitivity of the system, indeed the deviation is always inferior to $0.05 \%$ which may only be due to the formation of complexes having different type of hemocyanins as it was described before [6]. 


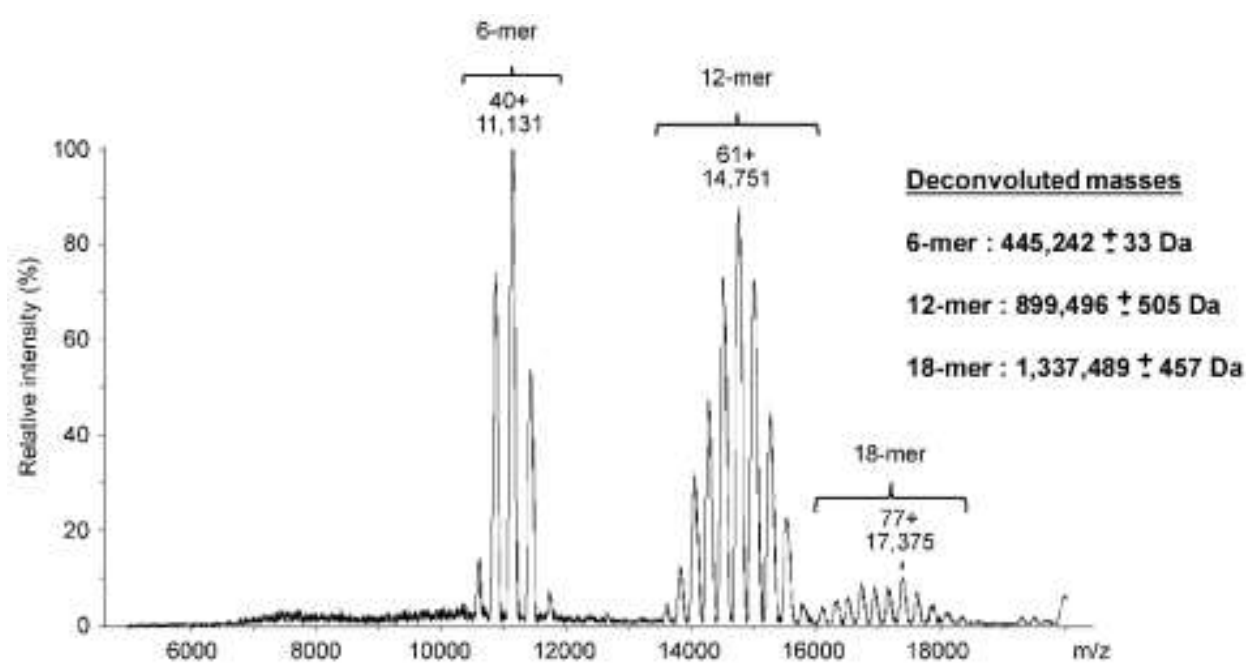

Fig 5. Hemocyanin mass spectra in native conditions. Experimental conditions : Hemocyanin $50 \mu \mathrm{M}$ approx. (in $20 \mathrm{mM}$ ammonium acetate ; $\mathrm{pH}=6.7$ ) ; bare fused silica capillary with porous tip (30 $\mu \mathrm{m}$ i.d.), flow rate $10 \mathrm{~nL} / \mathrm{min}$, capillary voltage $-1400 \mathrm{~V}$, dry gas $1.2 \mathrm{~L} / \mathrm{min}$, source temperature $140^{\circ} \mathrm{C}$.

The controlled flow rate delivered by the CE system permitted to generate a stable spray during the acquisition. Thereby the system exhibited a substantial robustness by enabling intensive infusion and extended acquisition duration. Results presented on this mass spectrum demonstrate the detection of the three forms of hemocyanin present in the sample. Those noncovalent assemblies have high molecular masses ranging from $445 \mathrm{kDa}$ to $1.34 \mathrm{MDa}$. Each complex could be structurally maintained with native conditions used and the ionization process delivered by the CESI prototype was suitable to profile those assemblies and especially the octodecamer hemocyanin are maintained by weak interaction and are typically difficult to ionize. To our knowledge, the only way to observe above $1 \mathrm{MDa}$ noncovalent complexes in native conditions is to use borosilicate capillaries. However the method developed in this work is the first one to be able to perform fully controlled and automated infusion in nanoESI and observe high molecular mass complexes above $1 \mathrm{MDa}$. One of the main improvement provided by this method, as compared to other nanoESI platforms, is its ease of use. The sample can be automatically infused 
into the MS without any other prior treatment of the capillary than a short washing. During the experiments reported here, no clogging of the capillary could be observed reinforcing the ease of use of this system while it is known to be an issue with other nanoESI platform especially when using high ionic strength buffers. Despise demonstrating the possibility to use the CESI interface to produce a nanoESI with a flow rate as low as $4 \mathrm{~nL} / \mathrm{min}$, characterization of the system demonstrated the possibility to obtained a fully controlled nanoESI flow rate. It is demonstrated that the developed method provides the ability to maintain the structure of noncovalent complexes throughout the nanoESI process to subsequently observe them in the MS.

Yet the utilization of the CESI prototype enabled the detection of the high molecular mass considered complexes with significant intensities. Those results prove the ability of the system, under the tested conditions, to maintain the existing interactions enabling the detection of noncovalent complexes, realizing the full potential of nanoESI a soft ionization process. Deconvoluted masses could be determined with an acceptable precision due to the detection, with an adequate resolution, of 5 to 10 different charge states depending on the considered complex. However buffer salt concentration was significant $(20 \mathrm{mM})$, the low flow rate at which the experiments were performed and the overall geometry of the interface provided a rather low background noise fully compatible with complexes detection. Intensity improvement, as compared to conventional ESI, are comparable to those obtained previously, the observed behavior being in agreement with the higher ionization efficiency reported using nanoESI [31]. The total volume of sample infused to perform an acquisition was about $74 \mathrm{~nL}$ which represent a 400 times reduction in terms of sample consumption as compared to standard ESI. This point 
is critical as the volume of hemolymph that can be extracted from Carcinus maenas is quite limited.

Like in the previous experiment, no clogging of the capillary could be observed during the experiment. The capillary was simply flushed after each experiment with buffer and could be directly used after again. A single capillary could be used for several days to infuse different samples without experimenting any significant variation of the mass spectra obtained.

\section{CONCLUSION}

In this study we have demonstrated the suitability of a prototype CESI-MS system to be used as a nanoESI infusion platform. This work was specifically design to assess the capabilities of the CESI prototype for the study through infusion of intact proteins and high molecular masses noncovalent assemblies. First, the sample injection device of the system was characterized and its capacity to hydrodynamically generate a stable electrospray with fully controlled flow rates ranging from less than $4 \mathrm{~nL} / \mathrm{min}$ to several hundreds of nanoliters per minute. The effect of the flow rate on the detection of an intact model protein under native conditions demonstrated an increased sensitivity when reducing the flow rate as a result of the reduction of ion suppression and increased ionization efficiency. Decreasing the flow rate from 456.9 to $13.7 \mathrm{~nL} / \mathrm{min}$ increased the sensitivity by a factor of 46 while the signal-to-noise ratio improved from 189.5 to 920.4 , this illustrating the ability of the system to be operated at very low flow rates to increase drastically sensitivity. Using the CESI interface as a nano electrospray emitter the study of $\beta$-ring under native conditions enabled the detection of the protein 
homodimer exhibiting a mass of $85.5 \mathrm{kDa}$. The study of the $\beta$-ring and P14 peptide complexes was then performed and showed the detection of two different proteinligand complexes demonstrating the suitability of the CESI interface for noncovalent complexes detection. In this particular case the stoichiometry of the complexes between the $\beta$-ring and the P14 peptide was determined. During the experiments the system exhibited high sensitivity, robustness and easy handling as no clogging could be observed. As a direct consequence of the very low flow rates used, the quantity of sample necessary to perform an acquisition could be drastically reduced with in the most favorable case a 400 fold reduction in comparison to conventional ESI. As the CESI prototype is designed to separative experiments, results demonstrated the flexibility of the system as it can be used to infuse sample into the MS. It opens new fields of applications such as stability constant determination especially with the affinity capillary electrophoresis hyphenised to MS. Finally the CESI prototype was used for the study of hemocyanin under native conditions. These experiments demonstrated the possibility to successfully detect high molecular masses complexes up to $1.34 \mathrm{MDa}$. This approach reveals the complete suitability and robustness of the CESI prototype as a nanospray emitter for the study of noncovalent complexes by MS. Additionally, the results obtained suggest that the utilization of this system could improve the quality of membrane protein studies as those typically require important ionic strength buffer while being available in scarce amounts.

\section{ACKNOWLEDGMENT}

Rabah Gahoual would like to thank the MRT for funding his Ph.D work. LSMIS would like to thank Beckman Coulter Inc. for the lend of a prototype capillary electrophoresis system and for their global support, and Bruker Daltonics Inc. for 
the loan of a MicroTOFQ MS. The authors would like to acknowledge Dr. Dominique Burnouf (IBMC, Strasbourg) for providing $\beta$ ring samples, and Dr. Frank Zal from Biological Station of Roscoff (France) for hemocyanin samples.

\section{REFERENCES}

(1) Loo JA (1997) Mass Spectrom. Rev. 16:1-23.

(2) Heck AJ, van den Heuvel RH (2004) Mass Spectrom. Rev. 23:368-389.

(3) Sharon M, Robinson CV (2007) Annu. Rev. Biochem. 76:167-193.

(4) Benesch JLP, Ruotolo BT, Simmons DA, Barrera NP, Morgner N, Wang L, Saibil HR, Robinson CV (2010) J. Struct. Bio. 172:161-168.

(5) Benesch JLP, Aquilina JA, Ruotolo BT, Sobott F, Robinson CV (2006) Chemistry \& Biology 13:597-605.

(6) Sanglier S, Leize E, Van Dorsselaer A (2003) J. Am. Soc. Mass Spectrom. 14:419429.

(7) Greig MJ, Gaus H, Cummins LL, Sasmor H, Griffey RH (1995) J. Am. Chem. Soc. 117:10765- 10766 .

(8) Hilton GR, Benesch JLP (2012) J. R. Soc. Interface 9:801-816.

(9) Constantopoulos TL, Jackson GS, Enke CG (1999) J Am Soc Mass Spectrom 10:625-634.

(10) Sterling HJ, Batchelor JD, Wemmer DE, Williams ER (2010) J Am Soc Mass Spectrom 21:1045-1049.

(11) Wilm MS, Mann M (1994) Int. J. Mass Spectrom. Ion Processes 136:167-180.

(12) Wilm MS, Mann M (1996) Anal. Chem. 68:1-8.

(13) Touboul D, Maillard L, Grässlin A, Moumne R, Seitz M, Robinson J, Zenobi R (2009) J. Am. Soc. Mass Spectrom. 20:303-311.

(14) Painter AJ, Jaya N, Basha E, Vierling E, Robinson CV, Benesch JLP (2008) Chemistry \& Biology 15:246-253.

(15) Moini M (2007) Anal. Chem. 79:4241-4246.

(16) Haselberg R, Ratnayake CK, de Jong GJ, Somsen GW (2010) J. Chromatogr. A 1217:7605-7611.

(17) Faserl K, Sarg B, Kremser L, Lindner H (2011) Anal. Chem. 83:7297-7305.

(18) Busnel JM, Schoenmaker B, Ramautar R, Carrasco-Pancorbo A, Ratnayake C, Feitelson JS, Chapman JD, Deelder AM, Mayoboroda OA (2010) Anal. Chem. 82:9476-9483.

(19) Heemskerk AAM, Busnel JM, Schoenmaker B, Derks RJE, Klychnikov O, Hensbergen PJ, Deelder AM, Mayboroda OA (2012) Anal. Chem. 84:4552-4559. 
(20) Wang Y, Fonslow BR, Wong CCL, Nakorchevsky A, Yates JR III (2012) Anal. Chem. 84:8505-8513.

(21) Zhu G, Sun L, Yan X, Dovichi NJ (2013) Anal. Chem. 85:2569-2573.

(22) Heemskerk AAM, Wuhrer M, Busnel JM, Koeleman CAM, Selman MHJ, Vidarsson G, Kapur R, Schoenmaker B, Derks RJE, Deelder AM, Mayboroda OA (2013) Electrophoresis 34:383-387.

(23) Gahoual R, Burr A, Busnel JM, Kuhn L, Hammann P, Beck A, François YN (2013) mAbs 5:479-490.

(24) Ramautar R, Busnel JM, Deelder AM, Mayoboroda OA (2012) Anal. Chem. $84: 885-892$

(25) Haselberg R, Harmsen S, Dolman MEM, de Jong GJ, Kok RJ, Somsen GW (2011) Anal. Chim. Acta 698:77-83.

(26) Haselberg R, de Jong GJ, Somsen GW (2013) Anal. Chem. 85:2289-2296.

(27) Wolff P, Olieric V, Briand JP, Chaloin O, Dejaegere A, Dumas P, Ennifar E, Guichard G, Wagner J, Burnouf DY (2011) J. Med. Chem. 54:4627-4637.

(28) Schmidt A, Karas M, Dülcks T (2003) J. Am. Soc. Mass Spectrom. 14:492-500.

(29) Burnouf DY, Olieric V, Wagner J, Fujii S, Reinbolt J, Fuchs RPP, Dumas P (2004) J. Mol. Biol. 335:1187-1197.

(30) Baclayon M, Shoemaker GK, Uetrecht C, Crawford SE, Estes MK, Venkataram Prasad BV, Heck AJR, Wuite GJL, Roos WH (2011) Nano Lett. 11:4865-4869.

(31) El-Faramawy A, Siu KWM, Thomson BA (2005) J. Am. Soc. Mass Spectrom. 16:1702-1707. 